PANORAMA

ISSN: $1909-7433$

ISSN: 2145-308X

ednorman@poligran.edu.co

Politécnico Grancolombiano

Colombia

\title{
TAREAS INTEGRADAS COMO ESTRATEGIA PARA EL DESARROLLO DE COMPETENCIAS ECONÓMICAS, FINANCIERAS Y CIUDADANAS DESDE EL ÁREA DE MATEMÁTICAS EN BÁSICA SECUNDARIA
}

Machado-Vidal, Marines Edith; Carrascal-Torres, Nohemy

TAREAS INTEGRADAS COMO ESTRATEGIA PARA EL DESARROLLO DE COMPETENCIAS ECONOMICAS,

FINANCIERAS Y CIUDADANAS DESDE EL ÁREA DE MATEMÁTICAS EN BÁSICA SECUNDARIA

PANORAMA, vol. 14, núm. 27, 2020

Politécnico Grancolombiano, Colombia

Disponible en: http://www.redalyc.org/articulo.oa?id=343964051010

DOI: https://doi.org/10.15765/pnrm.v14i27.1528

https://journal.poligran.edu.co/index.php/panorama/about/submissions\#copyrightNotice

Esta obra está bajo una Licencia Creative Commons Atribución-NoComercial-SinDerivar 4.0 Internacional. 
Artículos de investigación científica y tecnológica

PANORAMA, vol. 14, núm. 27, 2020

Politécnico Grancolombiano, Colombia

Recepción: 24 Octubre 2019

Aprobación: 05 Julio 2020

DOI: https://doi.org/10.15765/ pnrm.v14i27.1528

Redalyc: http://www.redalyc.org/ articulo.oa?id $=343964051010$

https://journal.poligran.edu.co/

index.php/panorama/about/

submissions\# copyrightNotice

CC BY-NC-ND
TAREAS INTEGRADAS COMO ESTRATEGIA PARA EL DESARROLLO DE COMPETENCIAS ECONÓMICAS, FINANCIERAS Y CIUDADANAS DESDE EL ÁREA DE MATEMÁTICAS EN BÁSICA SECUNDARIA

INTEGRATED TASKS AS A STRATEGY FOR THE DEVELOPMENT OF ECONOMIC, FINANCIAL AND CITIZENSHIP SKILLS FROM THE AREA OF MATHEMATICS IN BASIC SECONDARY

TAREAS INTEGRADAS COMO ESTRATEGIA

PARA EL DESARROLLO DE COMPETENCIAS ECONÓMICAS, FINANCIERAS Y CIUDADANAS DESDE EL ÁREA DE MATEMÁTICAS EN BÁSICA SECUNDARIA

Marines Edith Machado-Vidal marinesmavi@gmail.com Universidad de Córdoba, Colombia Nohemy Carrascal-Torres ncarrascal@gmail.com Universidad de Córdoba., Colombia

Resumen: El propósito del presente estudio fue evaluar la efectividad de la estrategia de tareas integradas desde el área de matemáticas en el desarrollo de las competencias económicas, financieras y ciudadanas en estudiantes de grado séptimo de la Institución Educativa los Garzones. El estudio tuvo un enfoque cuantitativo de tipo cuasiexperimental en el que se aplicó pretest y postest a dos grupos (control y experimental). El análisis incluyó la comparación de medias con t de Student para muestras relacionadas e independientes. La implementación de la estrategia mejoró significativamente las competencias, con lo cual los estudiantes pueden afrontar de una mejor manera situaciones de su entorno.

Palabras clave: Competencias económicas y financieras, competencias ciudadanas, tareas integradas, matemáticas.

Abstract: The purpose of this study was to evaluate the effectiveness of the strategy of integrated tasks from the area of mathematics in the development of economic, financial and citizen competencies in seventh grade students of the Institución Educativa Los Garzones. The study had a quasi-experimental quantitative approach in which pretest and postest were applied to two groups (control and experimental). Analysis included comparison of means with Student $t$ for related and independent samples. The implementation of the strategy significantly improved competencies, enabling students to better cope with situations in their environment. 
Keywords: Economic and financial competences, citizenship competence, integrated tasks, mathematics.

Resumo: O objetivo deste estudo foi avaliar a eficácia da estratégia de tarefas integradas da área de matemática no desenvolvimento de competências econômicas, financeiras e cívicas em alunos do sétimo ano da Instituição Educacional Los Garzones. O estudo teve uma abordagem quantitativa quase experimental na qual um pré-teste e um pósteste foram aplicados a dois grupos (controle e experimental). A análise incluiu a comparação das médias com $t$ de Student para amostras relacionadas e independentes. A implementação da estratégia melhorou significativamente as competências, com as quais os alunos podem enfrentar melhor as situações do seu ambiente.

\section{INTRODUCCIÓN}

El futuro de la educación representa un gran desafío que demanda de los gobiernos y de la comunidad educativa en general un esfuerzo por transformar los procesos de enseñanza y aprendizaje. Lo anterior se resume en la necesidad de construir nuevas realidades desde la escuela como escenario de formación por excelencia, en la cual convergen diversos fenómenos sociales, económicos y culturales que constituyen el rumbo de una sociedad.

Desde el escenario mundial hay un discurso que ha cobrado valor, especialmente entre los países miembros de organizaciones internacionales como la OCDE y la Comisión Económica para América Latina y el Caribe (CEPAL), las cuales consideran necesaria la formación en competencias económicas y financieras si se espera mejorar las condiciones de la región en los países que se hallan en vías de desarrollo; de esta manera se puede afirmar que:

Las competencias económicas y el emprendimiento pueden empoderar a los jóvenes para desarrollar actividades económicas intensivas en conocimiento, estimular la productividad y transformar la política de la región, facilitando la transición de la escuela al mundo del trabajo y dándoles herramientas para construir el futuro al que aspiran. (CEPAL, 2017, p.1)

Se debe resaltar, entonces, que no es posible pensar en el progreso conjunto de la población de una región sin la formación adecuada de sus ciudadanos en temas relacionados con lo económico, financiero y el impacto en su vida diaria, debido a que permiten que las personas sean capaces de tomar decisiones acertadas en situaciones socioeconómicas, así como reflexionar de manera crítica y tomar una postura determinante acerca de las políticas económicas que son promovidas desde el aspecto político-administrativo de los gobiernos de turno.

A pesar de lo anterior, la realidad de muchas de las escuelas colombianas parece ir en otra dirección, puesto que existe cierta apatía de los estudiantes hacia los números y las matemáticas en general, ya sea porque los temas les parecen complicados o no son de su interés, o producto de las metodologías tradicionales utilizadas por el docente, en las que los principales recursos didácticos son el marcador y el tablero. Dicha situación afecta el desarrollo del país, debido a que cada vez se presentan menos estudiantes para iniciar carreras relacionadas con las ciencias 
básicas, ingenierías, o cualquier otra en la que se haga uso intensivo de los números(Çevik \& Duman, 2018; Kong, 2018; Salim, Gunarhadi, \& Hidayatullah, 2020; Xie, Zou, Zhang, Wang, \& Kwan, 2019).

Evidencian el problema los resultados de Colombia en las pruebas Pisa, medición que se realiza en aquellos países que hacen parte de la OCDE. En el informe Pisa 2015 el país no sale bien librado; en matemáticas de 70 países participantes, Colombia ocupó el puesto 61, en el que obtuvo una puntuación de 350, muy por debajo de la media de los países participantes que fue de 490, e incluso mucho más lejos del puntaje de Singapur, que obtuvo un puntaje de 564 y logró el primer lugar (ICFES, 2016).

En un caso particular y atendiendo a las pruebas nacionales realizadas en Colombia, las cuales se reflejan en el Índice Sintético de Calidad (ISCE), también se pueden evidenciar los bajos resultados, ya que los estudiantes de grado $5^{\circ}$ de la Institución Educativa Los Garzones, aunque presentan una mejoría de un año a otro, todavía un gran porcentaje de ellos se ubican en nivel bajo (representado por el color rojo), en cuanto a las competencias matemáticas (ver gráfico 1 ).

\section{Matemáticas}
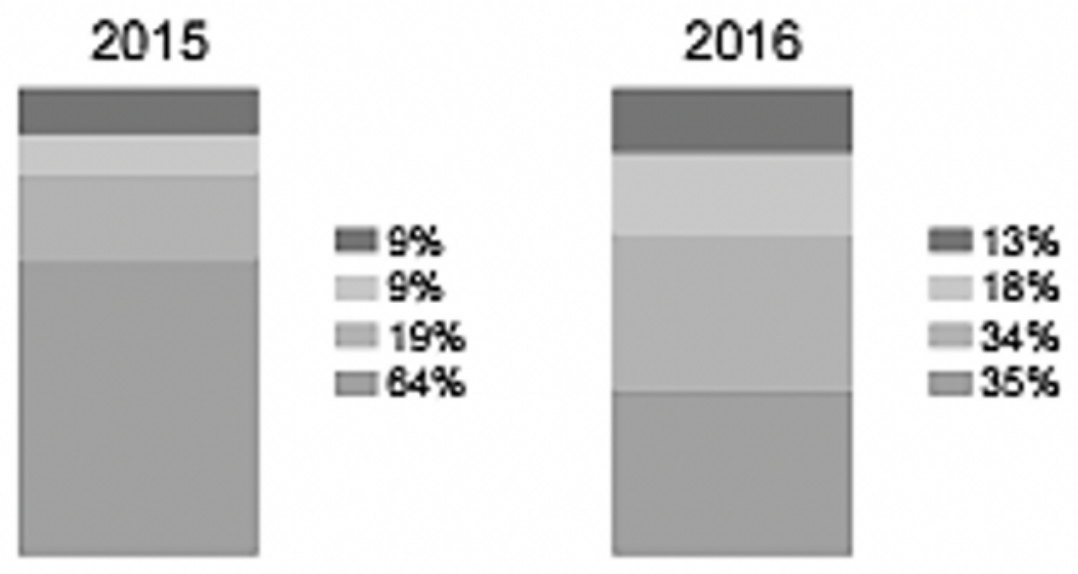

\section{Gráfico 1 .}

Resultados en matemáticas. Instegar 2015 y 2016.

Fuente: ISCE (2017).

Entre las estrategias planteadas para solventar los problemas descritos se destaca la creación en el año 2012 del Programa de Educación Económica y Financiera (PEEF), cuyo objetivo fundamental fue "fomentar el pensamiento crítico(Inostroza, 2015; León, Roca, \& Rius, 2018) en los estudiantes mediante el desarrollo de saberes, habilidades, actitudes y valores que les ayuden a reconocer, comprender, analizar y decidir frente a fenómenos económicos y financieros presentes en su cotidianidad" (MEN, 2013, p.1).

$\mathrm{Al}$ respecto se debe aclarar que la educación económica y financiera no tiene que ver solo con contenidos y programas de formación exclusivos de las matemáticas, sino que a partir de estas se pueden desarrollar otras competencias necesarias en los estudiantes, debido a que también se 
articulan, por ejemplo, con las competencias ciudadanas (Chaux, Lleras, \& Vela\#squez, 2004; Zambrano, 2018). Algunos estudios alrededor de este tema han demostrado que tanto las competencias matemáticas como las ciudadanas dialogan y convergen en la educación económica y financiera. Tanto así que algunos autores plantean que se debe fomentar la formación en valores cuando se maneja dinero: valores relacionados con el saber ser o saber valorar o saber comportarse: ser honesto, responsable, cumplidor y solidario (Ortega, 2017, p.10). En síntesis, las escuelas son las llamadas a desarrollar las estrategias de integración, para que desde la práctica pedagógica se pueda empoderar a los ciudadanos de estas competencias requeridas en el siglo XXI (Metlyakova \& Korobkova, 2012).

\section{MARCO TEÓRICO}

El marco teórico de esta investigación gira en torno a la educación económica y financiera y sus competencias. A continuación, se aborda cada una de estas categorías desde la visión de distintos autores:

\section{Educación económica y financiera}

Martínez (2012), afirma que la educación económica es una herramienta que permite enseñar a los niños y jóvenes a valorar las decisiones y consecuencias de su conducta y la conducta de los otros en un amplio rango de cuestiones, que deberían formar parte de la agenda de la educación personal y social de los niños y jóvenes. Estas cuestiones se relacionan con asuntos de su vida cotidiana que van desde las cartas a los santos reyes, el cuidado de los recursos naturales o personales, el robo y los intercambios justos. El autor hace especial énfasis en la formación de los niños y jóvenes como un aspecto fundamental que debe ser incluido en los planes de estudio y currículos escolares (Cooc, 2019).

Para profundizar en el tema se puede decir que la educación financiera comprende tres aspectos clave: primero, adquirir un conocimiento y una comprensión en materia de finanzas; segundo, desarrollar competencias en ese ámbito, es decir, tener capacidad para utilizar los conocimientos en beneficio propio; y tercero, ejercer la responsabilidad financiera, es decir, llevar a cabo una gestión adecuada de las finanzas personales, realizando elecciones informadas, con conocimiento de los riesgos asumidos (Domínguez, 2013, p.5).

También se debe mencionar que la educación económica y financiera se ha establecido como un elemento determinante en la formación de los educandos en Colombia, es por ello que desde el Ministerio de Educación Nacional se diseñó una guía de orientaciones pedagógicas para la educación económica y financiera (Bernal, Camacho, \& Martinez, 2013), la cual plantea diversos elementos que son considerados necesarios para formalizar la educación económica y financiera como proyecto pedagógico transversal(Ehm, Lindberg, \& Hasselhorn, 2014; Nicolás 
Arias-Velandia Giséle Eugénia Becerra-Plaza Ricardo José Salas-Díaz William Umar Rincón-Báez, Andrés Felipe Mejía-Rodríguez, 2018; Trujillo-Flórez et al., 2018; Vélez Rolón Manuel; Ramírez Triana, Carlos Ariel; Chicas Sierra, Sandra Milena; Mateus Tovar, Raúl Francisco; Martínez Amado, Rubén Darío; Pico Bonilla, Claudia Milena; Arias Suárez, Juan David; Cano Mejía, Vanessa; Ramos Barrera, M, Iván Francisco, 2019)

\section{Competencias financieras}

Siguiendo el enfoque del MEN acerca de la formación en economía y finanzas, se enfatiza el concepto de competencias generales en esta área como un proceso de "formación progresivo y continuo mediante el cual los estudiantes desarrollan las competencias necesarias para administrar los recursos y tomar decisiones responsables frente a las oportunidades y riesgos económicos y financieros que les permitan mejorar su calidad de vida" (MEN, 2014, p.40). La guía de orientaciones pedagógicas, emitida en el 2014, propone que las competencias enfatizan tres elementos que tienen que ver con la competencia cognitiva, procedimental y actitudinal, es decir, el saber, el saber hacer y el saber ser (ver tabla 1).

\begin{tabular}{lll}
\hline Competencia & Naturaleza o fin & Aspecto disciplinar/educación económica y financiera \\
\hline Cognitiva & Saber & Las y los estudiantes aprenden, proponen y desarrollan \\
Procedimental & Saber hacer & $\begin{array}{l}\text { proyectos que transforman realidades sociales y muestran } \\
\text { resultados concretos en el corto plazo. }\end{array}$ \\
\hline Actitudinal & Saber ser & Toma de decisiones financieras: las emociones que niñas, niños \\
& y adolescentes experimentan cuando se enfrentan a una \\
& situación.
\end{tabular}

\section{Tabla 1.}

Competencias cognitivas, procedimentales y actitudinales Fuente: Machado (2018), a partir de MEN (2014).

\section{Competencias ciudadanas en la educación económica y financiera}

Las competencias ciudadanas contribuyen a que las niñas, niños, adolescentes y jóvenes ejerzan su derecho a actuar como sujetos sociales activos de derechos para participar activa y responsablemente en las decisiones colectivas de manera democrática, resolver los conflictos en forma pacífica, respetar la diversidad cultural y proteger el medio ambiente, entre otros. Para Denegrí (2011), los ciudadanos deben manejar conceptos matemáticos básicos que les permitan desarrollar actividades relacionadas con su trabajo, negocio, administración de recursos, creación de empresa o para el consumo, entre otros aspectos de la vida diaria, que les ayuden a planificar sus gastos de forma oportuna y eficiente.

Otro de los elementos de las competencias ciudadanas desde la educación económica y financiera tiene que ver con el desarrollo de habilidades para relacionarse de manera asertiva en situaciones marcadas 
por este tipo de contenidos, por tal motivo el Ministerio de Educación Nacional (2014), ha propuesto en su guía de orientaciones pedagógicas que desde las competencias ciudadanas se aborde la competencia comunicativa, considerando que:

Las competencias comunicativas destacan el valor social del lenguaje y resaltan la importancia de interactuar activamente con la sociedad y participar en la transformación del mundo, relacionarse con sus congéneres desde una ética de la comunicación que propicia la diversidad, el encuentro y el diálogo de culturas, a la vez que se constituye en cimiento de la convivencia y del respeto, pilares de la formación ciudadana. (MEN, 2014, p.39)

\section{METODO}

Esta investigación estuvo enmarcada dentro del enfoque cuantitativo, ya que se utilizó la recolección y el análisis de datos para verificar hipótesis establecidas previamente. Teóricos mencionan que "el enfoque cuantitativo visto desde la dimensión metas de la investigación busca describir, explicar y predecir un fenómeno" (Hernández, Collado, \& Baptista, 2014, p.12). El paradigma fue empírico analítico, que, según Lopera et al., (2010) "es un camino para llegar a un resultado mediante la descomposición de un fenómeno en sus elementos constitutivos” (p.59).

Se eligió un diseño cuasiexperimental, dado que se trabajó con grupos intactos. El diseño se representa en la tabla 2:

$\begin{array}{lllll}\text { Control } & \text { Grupos } & \text { Pre-prueba } & \text { Tratamiento } & \text { Post-prueba } \\ \text { Prueba de equivalencia } & \mathrm{E} & \mathrm{O}_{1} & \mathrm{X} & \mathrm{O}_{2} \\ & \mathrm{C} & \mathrm{O}_{3} & ---- & \mathrm{O}_{4}\end{array}$

\section{Tabla 2.}

Diseño cuasiexperimental con grupo de comparación o control. Fuente: Hernández, Collado y Baptista (2014, p.13).

Nota. $\mathrm{E}=$ Grupo experimental, $\mathrm{C}=$ grupo de comparación, $\mathrm{X}=$ Tratamiento, $\mathrm{O}=$ evaluaciones (pre y post), la línea puntuada significa que hay dos grupos y no han sido formados por aleatorización, los cuales van a ser comparados.

En la tabla 2 se observa el diseño con grupo control (los grupos no han sido asignados al azar). Se realizó un pretest, que sirvió de diagnóstico para evaluar el estado inicial de los estudiantes, ya que lo ideal es que los grupos estuvieran en igualdad de condiciones experimentales antes de implementar la estrategia didáctica; seguidamente se aplicó la estrategia de tareas integradas para desarrollar las competencias económicas y financieras en los estudiantes. Y, por último, se aplicó un postest, que permitió determinar la efectividad de la estrategia aplicada.

Los participantes fueron dos grupos del grado séptimo de la Institución Educativa Los Garzones, de la ciudad de Montería. El grado Séptimo 1 con 22 estudiantes, y Séptimo 2 con 22 estudiantes. 
La investigación se llevó a cabo en cuatro etapas. En la etapa inicial o de diagnóstico se elaboraron las pruebas para evaluar el desarrollo de las competencias económicas, financieras y ciudadanas y se aplicó el pretest a ambos grupos. En la segunda etapa se diseñó la estrategia de tareas integradas, teniendo en cuenta un exhaustivo análisis teórico y de antecedentes. En la tercera etapa de aplicó la estrategia en el grupo experimental. En la cuarta etapa se aplicó el postest y se hizo el análisis de los resultados.

Los instrumentos utilizados fueron dos: pretest y postest, cada uno con 15 preguntas según el tema tratado en los módulos, todos los ítems basados en problemas para evaluar las competencias económicas, financieras y ciudadanas que utiliza el ICFES (2012-2016), y pruebas PISA. De igual forma, se incluyen ítems construidos por la docente de matemáticas. La confiabilidad y validez de los instrumentos se verificó mediante correlación de Pearson aplicado a los resultados de la prueba piloto y del juicio de expertos.

\section{RESULTADOS}

\section{Equivalencia entre grupos}

El análisis de los resultados del diagnóstico se hizo por grupos separados $\left(7^{\circ} 1\right.$ y $\left.7^{\circ} 2\right)$, utilizando para ello el software SPSS 21 versión libre. Primero se organizaron los datos en una tabla descriptiva, seguido se definió si los datos correspondían a una distribución normal, para proceder con pruebas paramétricas, en caso contrario, pruebas no paramétricas, por lo que se hizo necesario emplear la prueba de Shapiro-Wilk, que se aplicó para contrastar la hipótesis de normalidad de la población. El nivel de significancia o alfa tomado para realizar esta prueba fue de $5 \%$ de errores posibles en el momento de rechazar la hipótesis nula. A continuación, se compararon los grupos para establecer el grado de equivalencia entre ellos, se procedió con una prueba paramétrica $\mathrm{t}$ (de Student), para muestras independientes y relacionadas. Luego de las pruebas se concluyó que no existen diferencias significativas entre los grupos o, en otras palabras, que son grupos equivalentes.

\section{Resultados del diseño de la estrategia}

La estrategia de tareas integradas fue diseñada con el objetivo de desarrollar en los estudiantes de grado séptimo de básica secundaria las competencias económicas, financieras y ciudadanas. La aplicación se hizo durante el transcurso de ocho (8) semanas, correspondientes al tercer periodo académico del año 2017, en horas del área de matemáticas. Esta metodología colaborativa también estuvo orientada hacia el fortalecimiento del clima escolar.

Se diseñaron cuatro módulos referentes a habilidades cognitivas, procedimentales y actitudinales en cuanto a la planeación económica 
y financiera. También se evaluó la capacidad de prever obstáculos y consecuencias asociadas a las acciones o decisiones tomadas y administración de los recursos, abordando contenidos a partir de los contextos hogar (ahorro e inversión, valor y precio, tipos de crédito, tiempo, proyecto de vida, riesgo y recompensa, relación costo - beneficio), Estado (crecimiento y desarrollo, Producto Interno Bruto, impuestos y beneficios del gobierno, empleo y desempleo), empresa (factores de producción, oferta y demanda, costo de oportunidad, presupuesto, endeudamiento) y competencias ciudadanas (principios éticos y sociales).

Para el diseño se tomaron como puntos de partida los planteamientos de Morin (2010), por medio de los cuales se expone la importancia de establecer una relación de conocimiento entre el mundo real y los contenidos previstos para el aprendizaje. Desde esta perspectiva se plantearon actividades y ejercicios propios de la realidad económica y financiera de la familia colombiana, así como los distintos elementos que componen y constituyen la economía del país. De igual manera, los postulados de Denegrí (2011), y su tesis de la alfabetización económica son integrados a partir de los contenidos que se sugieren ser abordados de acuerdo con lo expuesto por el Ministerio de Educación Nacional (2014), en la edición del módulo de educación económica y financiera.

Las actividades y evaluaciones se plantearon de manera articulada como una forma de evaluar, mientras los estudiantes aprenden; se establecieron en el marco de una serie de contenidos, evaluaciones, actividades y/o ejercicios, por medio de los cuales se propiciaron espacios de aprendizaje, reflexión y apropiación de dichas competencias. En este aspecto se consideró fundamental establecer para las actividades grupales o individuales preguntas abiertas o ejercicio de desarrollo de ideas, construcción de argumentos, planteamiento de soluciones y estimación de ventajas y desventajas en situaciones específicas.

En la evaluación se emplearon preguntas cerradas, tipo ICFES, adicionalmente se ubicó un gráfico en forma de nube en el cual el estudiante debía demostrar cómo llegó a la respuesta. El diseño de la estrategia incluyó una guía del docente, correspondiente a la serie de sesiones de trabajo para cada uno de los módulos de educación económica, financiera, y ciudadana.

\section{Resultados del pretest grupo control - grupo experimental}

Para determinar el nivel de desarrollo de las competencias económicas y financieras y competencias ciudadanas que presentan los estudiantes de grado séptimo (grupo control y experimental), antes de la intervención se aplicó la evaluación pretest. Hay que recordar que esta evaluación estuvo basada en problemas tomados de las pruebas PISA, con un total de 15 ítems, ocho para la competencia económica y financiera y siete para la competencia ciudadana; además, la escala de medición es entre más ítems correctos mejor es el nivel de competencia. 
En el gráfico 2 se observa que ambos grupos tienen promedios muy parecidos, tanto en la competencia económica y financiera como en la ciudadana.

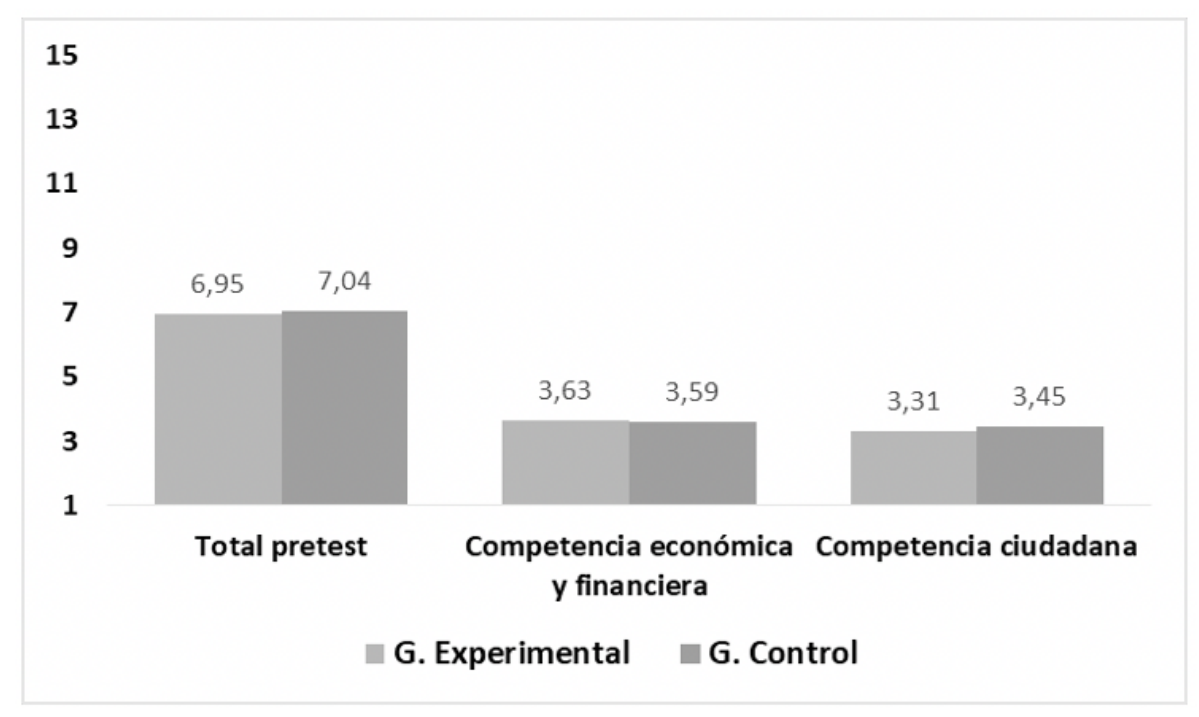

\section{Gráfico 2.}

Diagnóstico inicial

Fuente: Elaboración propia

Para complementar el análisis se usó la $\mathbf{t}$ de Student para muestras independientes, y los resultados se muestran en la tabla 3:

\begin{tabular}{|c|c|c|c|c|c|c|}
\hline & & \multicolumn{2}{|c|}{$\begin{array}{l}\text { Prueba de Levene de } \\
\text { igualdad de varianzas }\end{array}$} & \multicolumn{3}{|c|}{$\begin{array}{c}\text { prueba t para la igualdad } \\
\text { de medias }\end{array}$} \\
\hline & & $F$ & Sig. & $t$ & $\mathrm{gl}$ & $\begin{array}{c}\text { Sig. } \\
\text { (bilateral) }\end{array}$ \\
\hline $\begin{array}{l}\text { Competencia } \\
\text { económica y } \\
\text { financiera }\end{array}$ & $\begin{array}{l}\text { varianzas } \\
\text { iguales }\end{array}$ & ,076 & ,784 & 097 & 42 & ,924 \\
\hline $\begin{array}{l}\text { Competencia } \\
\text { ciudadana }\end{array}$ & $\begin{array}{l}\text { varianzas } \\
\text { iguales }\end{array}$ & ,334 & ,566 &, 267 & 42 & ,791 \\
\hline Total postest & $\begin{array}{l}\text { varianzas } \\
\text { iguales }\end{array}$ & ,329 & ,569 & $\begin{array}{c}- \\
, 117\end{array}$ & 42 & 908 \\
\hline
\end{tabular}

Tabla 3.

Prueba t de Student para muestras independientes pretest.

Fuente: SPSS

En la tabla 3 se puede observar que el P-valor (valor Sig), en todas las variables de estudio es mayor al nivel alfa $(0,05)$ (competencia financiera y económica es de $\mathbf{0 , 9 2 4 ; ~ c o m p e t e n c i a ~ c i u d a d a n a ~ e s ~ d e ~} \mathbf{0 , 7 9 1}$ y el total de

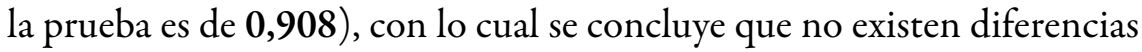
significativas entre los grupos. 
Para identificar el nivel de cada competencia se relaciona los puntajes del pretest con la escala de evaluación del colegio (ver tabla 4).

\begin{tabular}{|c|c|c|c|c|}
\hline Grupo & Variables & Promedio & $\begin{array}{c}\text { Calificación del } \\
\text { colegio }\end{array}$ & Nivel \\
\hline \multirow[t]{3}{*}{ Experimental } & $\begin{array}{l}\text { Competencia } \\
\text { económica y } \\
\text { financiera ( } 8 \text { ítems) }\end{array}$ & 3,63 & 2,3 & Bajo \\
\hline & $\begin{array}{l}\text { Competencia } \\
\text { ciudadana ( } 7 \text { items) }\end{array}$ & 3,31 & 2,1 & Bajo \\
\hline & $\begin{array}{l}\text { Total de la prueba } \\
\text { (15 items) }\end{array}$ & 6,95 & 2,2 & Bajo \\
\hline \multirow[t]{3}{*}{ Control } & $\begin{array}{l}\text { Competencia } \\
\text { económica y } \\
\text { financiera ( } 8 \text { ítems) }\end{array}$ & 3,5 & 2,3 & Bajo \\
\hline & $\begin{array}{l}\text { Competencia } \\
\text { ciudadana ( } 7 \text { ítems) }\end{array}$ & 3,4 & 2,2 & Bajo \\
\hline & $\begin{array}{l}\text { Total de la prueba } \\
\text { (15 items) }\end{array}$ & 7,04 & 2,3 & Bajo \\
\hline
\end{tabular}

\section{Tabla 4.}

Resultados evaluaciones en escala de calificación del colegio Fuente: Machado (2017)

Nota. Los resultados de las dimensiones están acomodados a la escala del colegio. $\mathrm{G}=$ grupos; $\mathrm{P}=$ promedio de ítems correctos; $\mathrm{C}=$ calificación del colegio; $\mathrm{N}=$ nivel; $\mathrm{BJ}=$ bajo; $\mathrm{BS}=$ básico; $\mathrm{AL}=$ alto; $\mathrm{SU}=$ superior.

\section{Resultados del postest grupo control - grupo experimental}

En el gráfico 3 se observa que hay mejorías en los promedios de ambos grupos, sin embargo, los resultados en el grupo experimental son mejores. 


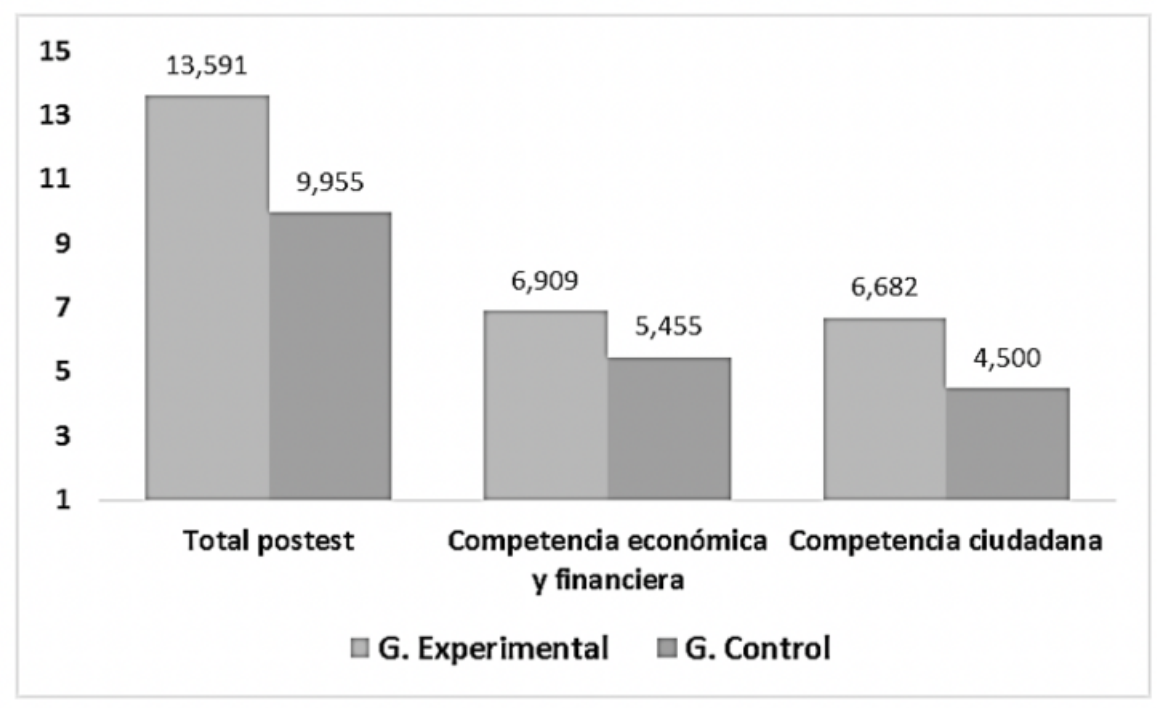

\section{Gráfico 3.}

Postest inter-grupo.

Fuente: Elaboración propia

Para complementar el análisis se usó la $\mathbf{t}$ de Student para muestras independientes y los resultados se muestran en la tabla 5 :

\begin{tabular}{|c|c|c|c|c|c|c|}
\hline & \multicolumn{2}{|c|}{$\begin{array}{l}\text { Prueba de Levene de } \\
\text { igualdad de varianzas }\end{array}$} & \multicolumn{3}{|c|}{$\begin{array}{c}\text { prueba t para la igualdad de } \\
\text { medias }\end{array}$} \\
\hline & & $F$ & Sig. & $t$ & $\mathrm{gl}$ & $\begin{array}{c}\text { Sig. } \\
\text { (bilateral) }\end{array}$ \\
\hline $\begin{array}{l}\text { Competencia } \\
\text { económica y } \\
\text { financiera }\end{array}$ & $\begin{array}{l}\text { varianzas } \\
\text { iguales }\end{array}$ & 3,464 & ,070 & 3,604 & 42 & ,001 \\
\hline $\begin{array}{l}\text { Competencia } \\
\text { ciudadana }\end{array}$ & $\begin{array}{l}\text { varianzas } \\
\text { iguales }\end{array}$ & 5,489 & ,060 & 8,255 & 42 & ,000 \\
\hline Total postest & $\begin{array}{l}\text { varianzas } \\
\text { iguales }\end{array}$ & 3,626 & ,064 & 7,729 & 42 & ,000 \\
\hline
\end{tabular}

Tabla 5.

Prueba $t$ de Student para muestras independientes postest Fuente: Machado (2018).

En la tabla 5 se puede observar que el P-valor que es el valor Sig en todas las variables de estudio es menor al nivel alfa $(0,05)$ (competencia financiera y económica es de $\mathbf{0 , 0 0 1}$; competencia ciudadana es de $\mathbf{0 , 0 0 0}$ y el total de la prueba es de $\mathbf{0 , 0 0 0}$ ) con lo cual se concluye que existen diferencias significativas entre los grupos.

Resultados pretest - postest grupo experimental

En el gráfico 4 se observa que hubo un aumento considerable en el promedio de ambas competencias en el grupo experimental, luego de la aplicación de la estrategia de las tareas integradas. 


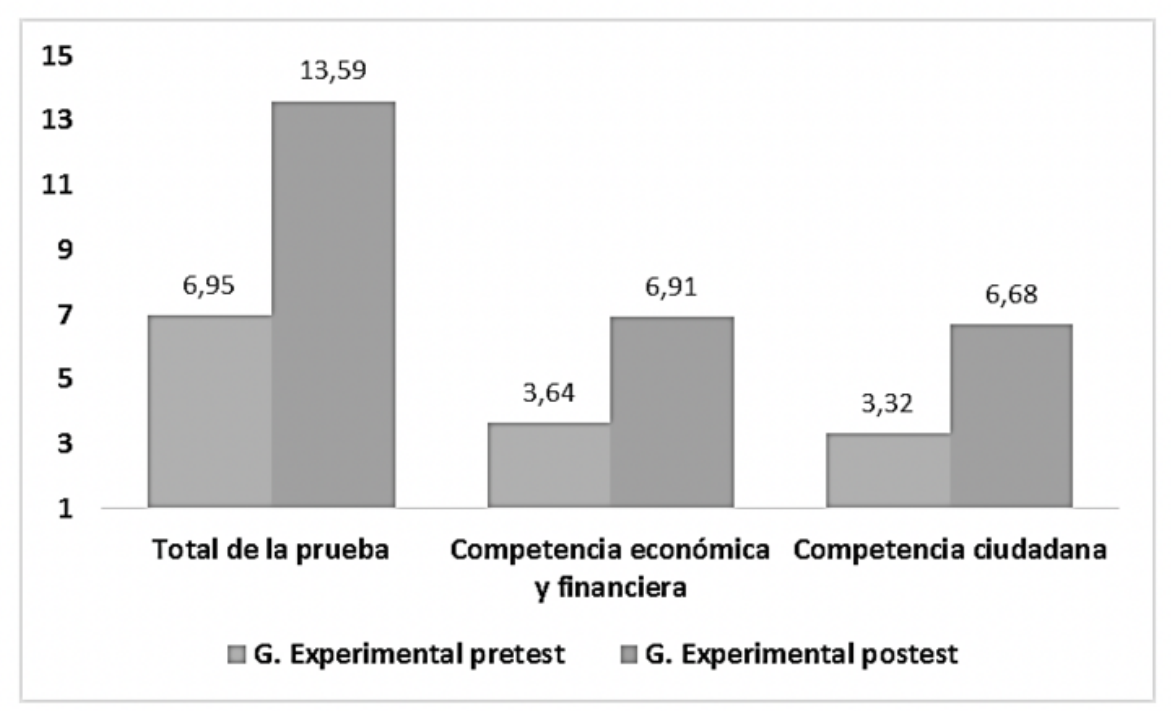

Gráfico 4.

Resultados postest intra-grupo

Fuente: Elaboración propia

Para complementar el análisis se usó la $\mathbf{t}$ de Student para muestras relacionadas, y los resultados se muestran en la tabla 6 :

\begin{tabular}{|l|l|c|c|c|}
\hline & Comparación intra-grupo experimental & $\mathbf{t}$ & $\mathbf{g l}$ & Sig. (bilateral) \\
\hline Par 1 & Competencia financiera y económica pretest - postest & $-6,821$ & 21 &, 000 \\
\hline Par 2 & Competencia ciudadana pretest - postest & $-10,115$ & 21 &, 000 \\
\hline Par 3 & Total de la prueba pretest - postest & $-11,529$ & 21 &, 000 \\
\hline
\end{tabular}

Tabla 6.

Prueba t de Student para muestras relacionadas Fuente: SPSS.

En la tabla 6 se puede observar que el P-valor (valor Sig) en todas las variables de estudio es menor al nivel alfa $(0,05)$ (competencia financiera y económica es de $\mathbf{0 , 0 0 0}$; competencia ciudadana es de $\mathbf{0 , 0 0 0}$ y el total de la prueba es de $\mathbf{0 , 0 0 0}$ ), con lo cual se concluye que existen diferencias significativas entre los resultados del grupo experimental antes y después de la aplicación de la estrategia de las tareas integradas.

Para identificar el nivel de cada competencia se relaciona los puntajes del pretest con la escala de evaluación del colegio (Ver tabla 7): 


\begin{tabular}{|c|c|c|c|c|}
\hline Grupo & Variables & Promedio & $\begin{array}{l}\text { Calificación } \\
\text { del colegio }\end{array}$ & Nivel \\
\hline \multirow{3}{*}{ Experimental } & $\begin{array}{l}\text { Competencia económica y financiera } \\
\text { (8 items) }\end{array}$ & 6,9 & 4,4 & Alto \\
\hline & $\begin{array}{l}\text { Competencia ciudadana } \\
\text { (7 items) }\end{array}$ & 6,6 & 4,8 & Superior \\
\hline & $\begin{array}{l}\text { Total de la prueba } \\
\text { (15 items) }\end{array}$ & 13,59 & 4,6 & Alto \\
\hline \multirow{3}{*}{ Control } & $\begin{array}{l}\text { Competencia económica y financiera } \\
\text { (8 items) }\end{array}$ & 5,4 & 3,4 & Básico \\
\hline & $\begin{array}{l}\text { Competencia ciudadana } \\
\text { ( } 7 \text { ítems) }\end{array}$ & 4,5 & 3,3 & Básico \\
\hline & $\begin{array}{l}\text { Total de la prueba } \\
\text { (15 ítems) }\end{array}$ & 9,9 & 3,2 & Básico \\
\hline
\end{tabular}

Tabla 7.

Resultados evaluación postest en escala de calificación del colegio Fuente: Machado (2018).

En la tabla 7 se hace evidente el aumento en los niveles de desarrollo de las competencias luego de la intervención con la estrategia de tareas integradas en el grupo experimental. Antes del tratamiento los resultados eran bajos en todas las variables; después de la intervención la competencia económica y financiera pasó a un nivel alto, y la competencia ciudadana a un nivel superior.

\section{DISCUSIÓN}

Los resultados del pretest evidencian que ambos grupos, tanto el experimental como el control $\left(7^{\circ} 1\right.$ y $\left.7^{\circ} 2\right)$, se ubican en nivel bajo en el desarrollo de las competencias económicas, financieras y ciudadanas. Según Quintero (2014), los resultados bajos en cuanto a las competencias estudiadas se deben a que los contenidos son relativamente nuevos y, además, no existen lineamientos claros para abordarlas en el aula. También la dinámica del docente es un factor influyente, por cuanto no ha dejado de ser el protagonista en el proceso de enseñanza-aprendizaje, contrario a una educación constructivista. Al respecto, Chuquisana (2015) menciona que "se debe dejar de lado la enseñanza tradicional que proponía empezar un nuevo tema de matemática a partir de las fórmulas. Los temas de matemática financiera deben ser abordados a partir de la resolución de problemas contextualizados" (p.110).

Los resultados de la prueba indican que hay dificultad al momento de identificar las formas básicas para manejar los recursos económicos de la manera más eficiente; el problema evidente podría tener sus causas debido a la falta programática y de temas en relación con la competencia económica y financiera. En cuanto a la competencia ciudadana, las dificultades están en la falta de integración social en la formación, teniendo en cuenta que los estudiantes trabajan muy poco en grupo, algunas veces son irrespetuosos con sus compañeros, también se observa 
exclusión en muchos aspectos, no les gusta seguir normas, entre otros factores determinantes en la convivencia en sociedad.

Luego de aplicar el postest se puede afirmar que los estudiantes del grupo control, en el cual se desarrollaron las clases de una forma tradicional, aumentaron el nivel de desarrollo de la competencia, pero en menor proporción que los estudiantes del grupo experimental. Los alumnos del grupo control fueron receptores pasivos y memorísticos. Por otra parte, en el grupo experimental fue notorio el cambio en los aspectos cognitivo, procedimental y actitudinal. Los estudiantes de dicho grupo fueron aumentando su nivel tras el desarrollo de las actividades en el marco de la estrategia de las tareas integradas; se notó el cambio, la responsabilidad, el desarrollo de la curiosidad y la capacidad de crear para solucionar los problemas económicos y financieros. En términos generales, se obtuvieron las ventajas de trabajar con el enfoque en competencias, como lo afirman algunos teóricos (Andrade et al., 2015; Chuquisana, 2015; Tejeda, 2009), que exponen cómo el enfoque centrado en el desarrollo de la competencias económicas y financieras desarrolla también las competencias ciudadanas.

\section{CONCLUSIONES}

Los resultados obtenidos en el estudio permitieron verificar que la implementación de la Estrategia Tareas Integradas (E.T.I), desde el área de matemáticas, mejora el nivel de desarrollo de la competencia económica, financiera y ciudadana de los estudiantes de grado séptimo de la Institución Educativa los Garzones.

Los resultados del pretest indicaron que no existían diferencias significativas entre los grupos antes de la intervención y al convertir dichos datos a la escala que se maneja en la institución educativa; el nivel predominante en las competencias fue el bajo, lo cual quedó evidenciado en la dificultad que tenían los estudiantes para manejar recursos, tomar decisiones financieras y solucionar conflictos.

La apropiación teórica-conceptual aportó valiosa información que permitió el adecuado diseño de la Estrategia Tareas Integradas, de la cual quedó como producto una guía con cuatro módulos de trabajo de las competencias económicas, financieras y ciudadanas para las clases de matemáticas.

Con la implementación de la estrategia se logró que los estudiantes y el docente estuvieran más motivados, hubo trabajo colaborativo y autónomo, se adaptaron fácilmente, se desarrolló la evaluación por competencia y se valoró el proceso. Además, los alumnos aprendieron por descubrimiento (Bruner, 1961), lo cual se evidenció cuando buscaron información, organizaron, tabularon datos y mostraron resultados.

Los resultados intergrupo obtenidos en el postest mostraron que ambos grupos aumentaron su promedio, pero el grupo experimental logró mejores puntuaciones.

En el análisis intragrupo del grupo experimental, se observa un aumento considerable de los promedios, lo cual evidencia que hubo un 
aumento significativo en el desarrollo de las competencias, análisis que fue corroborado con la prueba estadística t de Student.

Con esta investigación se fomenta el cambio de los paradigmas actuales de enseñanza, hacia otro que contemple la creación de experiencias por medio de la intervención educativa, al igual que los estudios de Aguirre (2015) y Ortega (2017), los cuales concluyen que el desarrollo de competencias no es fácil, por lo que el docente debe planear y establecer metodologías facilitadoras que conlleven a una formación integral. En este trabajo, el docente diseñó una guía que contempló estrategias didácticas innovadoras que propiciaron aprendizajes significativos.

Otro de los logros de la estrategia fue el empoderamiento de términos económicos y financieros, puesto que luego de realizar el trabajo práctico el estudiante logra descubrir dichos conceptos, como un proceso a posteriori que da relevancia y fundamento a lo que se ha logrado desde los ejercicios procedimentales. Enlazar el concepto con la práctica es quizás el momento de mayor aprendizaje para el estudiante, que encuentra a su vez la aplicabilidad y coherencia a sus acciones económicas y financieras.

\section{REFERENCIAS}

Aguirre, C. (2015). Modelo curricular de educación financiera para grados sexto y séptimo (tesis para obtener el título de Magíster en Enseñanza de las Ciencias exactas y naturales, Universidad Nacional de Colombia-Sede Manizales). Recuperado de http://www.bdigital.unal.edu.co/50500/1/1 0259601.2015.pdf

Arias-Velandia, N. Becerra-Plaza, GE. Salas-Díaz, R. (2018). Retos a los programas de administración una mirada al saber. Resultados de la evaluación externa (2012-2016). Bogotá: Institución Universitaria Politécnico Grancolombiano.

Bernal, M. A., Camacho, F. J., \& Martinez, R. (2013). Dimensional analysis and Rutherford scattering. European Journal of Physics, 34(1), L5-L8. https: //doi.org/10.1088/0143-0807/34/1/L5

Bruner, J. (1961). The act of discovery. Harvard educational review, 31, 21-32.

CEPAL. (2017). La inversión extranjera directa en América Latina y el Caribe.

Çevik, H., \& Duman, T. (2018). Analyzing the effects of E-learning on science education. International Journal of Instruction, 11(1), 345-362. https:// doi.org/10.12973/iji.2018.11124a

Chaux, E., Lleras, J., \& Vela\#squez, A. M. (2004). Competencias ciudadanas de los estándares al aula: una propuesta de integración a las áreas académicas. Bogotá: Ediciones Uniandes.

Chuquisana, F. (2015). Matemática financiera en la escuela secundaria para la alfabetización financiera y la formación ciudadana: una propuesta para la formación de profesores en temas de interés simple y compuesto. Recuperado de http://tesis.pucp.edu.pe/repositorio/bitstream/handle/123456789/6 406/CHUQUISANA_MORA_FREDDY_MATEMATICA_FINAN CIERA.pdf?sequence $=1$

Cooc, N. (2019). Teaching students with special needs: International trends in school capacity and the need for teacher professional development. 
Teaching and Teacher Education, 83, 27-41. https://doi.org/10.1016/j. tate.2019.03.021

Denegrí, M., Sepúlveda, J., \& Godoy, M. (2011). Actitudes hacia la compra y el consumo de estudiantes de pedagogía y profesores en ejercicio en Chile. Revista Psicología desde el Caribe, (28).

Domínguez, J. (2013). Educación Financiera para Jóvenes: una visión introductoria. Instituto universitario de análisis económico y social. Universidad de Alcalá (No. 05/13). Recuperado de http://www3.uah.es/ iaes/publicaciones/DT_05_13.pdf

Ehm, J.-H., Lindberg, S., \& Hasselhorn, M. (2014). Reading, writing, and math self-concept in elementary school children: Influence of dimensional comparison processes. European Journal of Psychology of Education, 29(2), 277-294. https://doi.org/10.1007/s10212-013-0198-x

Hernández, R., Fernández, C., \& Baptista, P. (2014). Metodología de la investigación. México: Editorial Mc Graw Hill.

ICFES. (2016). Resumen Ejecutivo Colombia PISA 2015. Recuperado de: www.icfes.gov.co/docman/.../2785-informe-resumenejecutivo-colombia-en-pisa-2015

ICFES. (2017). Guia de orientación saber $3 .^{\circ}, 5^{\circ}$ y 9.․ Bogotá: Recuperado de http://www.icfes.gov.co/estudiantes-y-padres/pruebas-saber-3-5-y-9estudiantes.

Inostroza, F. A. (2015). Análisis crítico del discurso sobre la noción de estudiante con dificultades del aprendizaje en el marco de un proyecto de integración escolar en una escuela municipal de Chile. Revista Mexicana de Investigacion Educativa, 20(66), 941-958. Retrieved from https://www.scopus.com/inward/record.uri?eid=2-s2.0-84939530 611\&partnerID $=40 \& \mathrm{md} 5=\mathrm{cafa} 8716 \mathrm{e} 67 \mathrm{~b} 5 \mathrm{a} 5 \mathrm{ac} 0 \mathrm{~b} 5433 \mathrm{~cd} 8 \mathrm{c} 9 \mathrm{fa} 1 \mathrm{e}$

Kong, S.-C. (2018). Parents' perceptions of e-learning in school education: implications for the partnership between schools and parents. Technology, Pedagogy and Education, 27(1), 15-31. https://doi.org/10. 1080/1475939X.2017.1317659

León, P. M., Roca, J. B., \& Rius, N. I. (2018). Quasi-Experimental Design to Analysis Critical Thought in Secondary . Revista Electronica de Investigacion Educativa, 20(4), 123-132. https://doi.org/10.24320/redi e.2018.20.4.1705

Martínez, E. (2012). La alfabetización socioeconómica y financiera y la educación para el consumo sostenible en México: algunas reflexiones desde la psicología y la educación. CPU-e, Revista de Investigación Educativa, (8), 79-93. Recuperado de: file://C:/Users/ASUS/Downloads/85-473-1PB.pdf

MEN. (2014). Orientacionespedagógicas para la educación económica y financiera.

Metlyakova, L. A., \& Korobkova, V. V. (2012). Integration of formal and nonformal education of children and adults in institutions of supplementary education as a factor of social and pedagogical support for families in difficult situations. World Applied Sciences Journal, 20(SPL.ISS.), 51-57. https://doi.org/10.5829/idosi.wasj.2012.20.10011

Morín, E. (2010). Sobre la interdisciplinariedad. Publicaciones Icesi (62).

Ortega, A. (2017). Aprendizaje basado en problemas como estrategia para el desarrollo de la competencia económica y financiera desde la enseñanza del álgebra en grado octavo. Universidad de Córdoba, Colombia, 3er Simposio 
Internacional y 4to Coloquio Regional de Investigación Educativa y Pedagógica.

Quintero, S. (2014). Educación económica y financiera en Colombia desde la perspectiva del desarrollo humano (Tesis doctoral). Universidad Santo Tomás, Bogotá. Recuperado de: https://repository. usta. edu. co/ bitstream/handle/11634/3399/Quinterosigifredo2014. pdf).

Salim, A., Gunarhadi, \& Hidayatullah, M. F. (2020). Measuring belief-based perception towards children with special needs in inclusive education. Universal Journal of Educational Research, 8(3D), 19-25. https://doi.or g/10.13189/ujer.2020.081704

Trujillo-Flórez, L. M., Martínez Contreras, R. M., Espitia López, H., Rojas Paredes, J. C., Vargas Leguizamón, Y. R., \& Castro Cabal, G. A. (2018). Experiencias de innovación educativa (1st ed.; L. M. Flórez-Trujillo, Ed.). Bogotá: Politécnico Grancolombiano.

Vélez Rolón Manuel; Ramírez Triana, Carlos Ariel; Chicas Sierra, Sandra Milena; Mateus Tovar, Raúl Francisco; Martínez Amado, Rubén Darío; Pico Bonilla, Claudia Milena; Arias Suárez, Juan David; Cano Mejía, Vanessa; Ramos Barrera, M, Iván Francisco, A. M. P. (2019). Experiencias de aprendizaje significativo para la apropiación de conocimientos en ciencias económicas, administrativas y contables (1st ed.; P.-B. C. Milena, Ed.). Bogotá: Institución Universitaria Politécnico Grancolombiano.

Xie, H., Zou, D., Zhang, R., Wang, M., \& Kwan, R. (2019). Personalized word learning for university students: a profile-based method for e-learning systems. Journal of Computing in Higher Education, 31(2), 273-289. ht tps://doi.org/10.1007/s12528-019-09215-0

Zambrano, E. L. (2018). Prácticas pedagógicas para el desarrollo de competencias ciudadanas. Revista Electronica de Investigacion Educativa, 20(1), 69-82. https://doi.org/10.24320/redie.2018.20.1.1409

\section{Información adicional}

Para citar este artículo: / To cite this article: / Para citar este artigo:: Machado-Vidal, M., \& Carrascal-Torres, N. (2020). TAREAS INTEGRADAS COMO ESTRATEGIA PARA EL DESARROLLO DE COMPETENCIAS ECONÓMICAS, FINANCIERAS Y CIUDADANAS DESDE EL ÁREA DE MATEMÁTICAS EN BÁSICA SECUNDARIA. (P. Grancolombiano, Ed.). Revista Panorama, 14(27), DOI: http://dx.doi.org/10.15765/ pnrm.v14i27.1528 